\title{
Using modified Gaussian distribution to study the physical properties of one and two-component ultracold atoms
}

\author{
Chou-Chun Huang and Wen-Chin Wu \\ Department of Physics, National Taiwan Normal Univesity, Taipei 11650, Taiwan
}

(Dated: November 15, 2018)

\begin{abstract}
Gaussian distribution is commonly used as a good approximation to study the trapped onecomponent Bose-condensed atoms with relatively small nonlinear effect. It is not adequate in dealing with the one-component system of large nonlinear effect, nor the two-component system where phase separation exists. We propose a modified Gaussian distribution which is more effective when dealing with the one-component system with relatively large nonlinear terms as well as the twocomponent system. The modified Gaussian is also used to study the breathing modes of the twocomponent system, which shows a drastic change in the mode dispersion at the occurrence of the phase separation. The results obtained are in agreement with other numerical results.
\end{abstract}

PACS numbers: 03.75.Hh, 32.80.Pj, 03.65.-w

\section{INTRODUCTION}

Single-component Bose-Einstein condensate (BEC) in trapped atoms has been observed more than ten years ago. Since then, in particular in recent years, laser cooling technique has been extended to trap atoms of multiple components. The basic technique is called "sympathetic cooling" that cools the second kind of atoms by means of the first kind pre-cooled atoms. Various combinations of two-component atomic gas have been mixed and cooled. The first was done on the mixture of the same kind of bosonic atoms with different hyperfine spin states. For instance, the combinations of ${ }^{87} \mathrm{Rb}|2,2\rangle$ and ${ }^{87} \mathrm{Rb}|1,-1\rangle$ states [1], and of ${ }^{87} \mathrm{Rb}|2,1\rangle$ and ${ }^{87} \mathrm{Rb}|1,-1\rangle$ states [2]. Soon after, the experiment has been extended to cool the mixture of different kinds of bosons. This includes the mixture of ${ }^{87} \mathrm{Rb}$ and ${ }^{41} \mathrm{~K}$ atoms [3] and ${ }^{133} \mathrm{Cs}$ and ${ }^{87} \mathrm{Rb}$ atoms [4]. The mixtures of bosons and fermions have also been achieved, such as the combination of ${ }^{7} \mathrm{Li}$ and ${ }^{6} \mathrm{Li}$ atoms [5, 6], ${ }^{6} \mathrm{Li}$ and ${ }^{23} \mathrm{Na}$ atoms [7], and ${ }^{40} \mathrm{~K}$ and ${ }^{87} \mathrm{Rb}$ atoms 8 . The fermion-fermion mixtures have also been accomplished, such as the combinations of two different spin states in ${ }^{40} \mathrm{~K}$ atoms [9] and in ${ }^{6} \mathrm{Li}$ atoms 10, 11]. The latter has led to the formation of bosonic molecules using Feshbach resonance and has advanced the observation of BEC-BCS crossover [12, 13, 14]. More recently, imbalanced spin population in fermion-fermion mixture has also been created and boosted researches on these systems [15, 16].

In the literature, there exists theoretical works on equilibrium and dynamical properties of the two-component cold atoms, including the investigations on the bosonboson mixtures [17, 18, 19, 20, 21] and the fermionfermion mixtures [22, 23]. For two-component bosonboson mixtures, in which both components are Bose condensed, there are two major theoretical approaches. One is concerned with the Thomas-Fermi approximation (TFA), in which kinetic-energy terms are ignored. The other starts with the coupled Gross-Pitaveskii (GP) equations, which are then solved numerically. In this pa- per, we attempt an alternative approach to investigate the two-component system based on variational method. Through the introduction of a modified Gaussain trial function, one is able to study the condition of the system being in the state of phase separation or miscible. The breathing modes of the system is also studied and shown to be intimately connected to their ground-state behavior. As a matter of fact, the modified Gaussian distribution is clearly shown to be a better description than the simple Gaussian even to the one-component trapped $\mathrm{BEC}$, especially when the nonlinear effect is more important. Variational method in adjunction with the introduced trial modified Gaussian function can be easily generalized to study the two-component boson-fermion and fermion-fermion mixtures. The latter includes the system of imbalanced spin population of current interest.

The paper is organized as follows. In Sec. II we first give the Hamiltonian of a two-component boson-boson mixture. Coupled Gross-Pitaveskii energy functionals are derived for the system assuming that both components are fully Bose condensed. A subsection is devoted to sketch the variational method. In Sec. III] we introduce a trial "modified Gaussian function" whose groundstate behaviors to the one-component Bose system are compared in details with those of the simple Gaussian distribution and the TFA. In Sec. IV] the trial modified Gaussian function are applied to study the equilibrium and dynamical properties of the two-component Bose system, and in Sec. V] a brief conclusion is given. In the current context, we focus on the trapped system of boson-boson mixture. Similar approaches to the fermionboson and fermion-fermion mixtures will be possible future works. 


\section{FORMALISM AND METHODOLOGY}

\section{A. Coupled GP Energy Functionals}

For a trapped two-component boson-boson mixture, the Hamiltonian can be given by

$$
H=H_{1}+H_{2}+H_{12},
$$

where

$$
\begin{aligned}
H_{i} & =\int d \mathbf{r} \phi_{i}^{\dagger}(\mathbf{r})\left[-\frac{\hbar^{2} \nabla^{2}}{2 m_{i}}+V_{i}(\mathbf{r})-\mu_{i}\right] \phi_{i}(\mathbf{r}) \\
& +\frac{g_{i i}}{2} \iint d \mathbf{r} d \mathbf{r} / \phi_{i}^{\dagger}(\mathbf{r}) \phi_{i}^{\dagger}\left(\mathbf{r}^{\prime}\right) \delta\left(\mathbf{r}-\mathbf{r}^{\prime}\right) \phi_{i}\left(\mathbf{r}^{\prime}\right) \phi_{i}(\mathbf{r})(2)
\end{aligned}
$$

and

$$
\left.H_{12}=g_{12} \iint d \mathbf{r} d \mathbf{r}^{\prime} \phi_{1}^{\dagger}(\mathbf{r}) \phi_{2}^{\dagger}\left(\mathbf{r}^{\prime}\right) \delta\left(\mathbf{r}-\mathbf{r}^{\prime}\right) \phi_{2}\left(\mathbf{r}^{\prime}\right) \phi_{1}(\mathbf{r}\rceil 3\right)
$$

Here $i=1,2$ denote the species $i$ of the mixture, $\phi_{i}^{\dagger}(\mathbf{r})$ and $\phi_{i}(\mathbf{r})$ are creation and annihilation operators on the particle of species $i$ at $\mathbf{r}$, and $\mu_{i}$ denote the chemical potential of the species $i$. The entire system is trapped in an isotropic magnetic potential $V_{i}(\mathbf{r})=\frac{1}{2} m_{i} \omega_{i}^{2} r^{2}$ respectively for each component $i$. It is assumed that the interaction is dominated by the $s$-wave approximation in the dilute limit. Consequently the interaction coupling constants in (2) and (3) can be written in a unified way

$$
g_{i j}=\frac{4 \pi \hbar^{2} a_{i j}}{m_{i j}}
$$

where $a_{i j}$ is the scattering length for a particle in species $i$ with a particle in species $j$. The "mass" $m_{i j} \equiv$ $2 m_{i} m_{j} /\left(m_{i}+m_{j}\right)$ with $m_{i}$ the bare mass of the particle in species $i$. For simplicity, we shall denote $g_{11} \equiv g_{1}$; $g_{22} \equiv g_{2}$ and $a_{11} \equiv a_{1} ; a_{22} \equiv a_{2}$ afterwards.

At sufficiently low temperatures, consider that both components are fully Bose condensed, the thermal average of $H_{i}$ in (2) then reduces to the Gross-Pitaveskii (GP) energy functional:

$$
\begin{aligned}
E_{i}=\left\langle H_{i}\right\rangle & =\int d \mathbf{r} \Phi_{i}^{*}(\mathbf{r})\left[-\frac{\hbar^{2} \nabla^{2}}{2 m_{i}}+\frac{1}{2} m_{i} \omega_{i}^{2} r^{2}-\mu_{i}\right. \\
& \left.+\frac{g_{i}}{2}\left|\Phi_{i}(\mathbf{r})\right|^{2}\right] \Phi_{i}(\mathbf{r})
\end{aligned}
$$

where $\Phi_{i}=\left\langle\phi_{i}\right\rangle$. Similarly the thermal average of $H_{12}$ in (3) reduces to

$$
V_{12}=\left\langle H_{12}\right\rangle=g_{12} \int d \mathbf{r}\left|\Phi_{1}(\mathbf{r})\right|^{2}\left|\Phi_{2}(\mathbf{r})\right|^{2}
$$

\section{B. Variational Method}

One major goal of this paper is to apply the variational method to investigate the dynamics of the coupled twocomponent ultracold atoms. The key to the variational approach is to first look for a trial wave function $\Phi_{i}(\mathbf{r})$ for each component $i$, which is reasonable to the ground state of the system. With these trial wave functions, one then incorporates appropriate dynamical variables $\left[\Phi_{i}(\mathbf{r}) \rightarrow \Phi_{i}(\mathbf{r}, t)\right]$, which are sensible to the dynamics under studies. The next step is to derive the corresponding Lagrangian: $L=\int d \mathbf{r} T-E$ using $\Phi_{i}(\mathbf{r}, t)$. Here

$$
T=\sum_{i=1,2} \frac{i \hbar}{2}\left(\Phi_{i}^{*} \frac{\partial \Phi_{i}}{\partial t}-\Phi_{i} \frac{\partial \Phi_{i}^{*}}{\partial t}\right)
$$

and

$$
E=\langle H\rangle=E_{1}+E_{2}+V_{12},
$$

where $E_{1}, E_{2}$, and $V_{12}$ are calculated based on (5) and (6) with $\Phi_{i}(\mathbf{r})$ being replaced by $\Phi_{i}(\mathbf{r}, t)$ now. Finally a set of coupled dynamical equations of motion can be derived through the Euler-Lagrange equations:

$$
\frac{\partial L}{\partial \beta}=\frac{d}{d t} \frac{\partial L}{\partial \dot{\beta}}
$$

where $\beta$ is any one of the dynamical variables chosen. The corresponding dynamics, in particular the collective mode dispersion relation, can be obtained by solving the roots of the dynamical matrix (diagonalization).

\section{TRIAL WAVE FUNCTION}

Before a proper trial wave function is introduced for each species in the trapped two-component cold atoms, it is useful to first examine the validity of the simple Gaussian distribution.

\section{A. Remarks: Gaussian Distribution}

When temperature approaches zero, single-component trapped Bose condensate (isotropic case) is governed by the GP energy functional given in Eq. (5). [In this case the index $i$ in (5) is redundant and is thus removed.] When the coupling $g$ is infinitesimally small, the ground state of the system is perfectly a Gaussian distribution:

$$
\Phi(\mathbf{r})=C \exp \left(-A r^{2}\right) .
$$

Gaussian function is "non-perturbative" and has the advantage of easily integrated over the space. When $g$ starts to increase, however, a Gaussian may no longer be a good one to describe the system. In the limit of 
large $g$, Gaussian distribution fails and the most common approximation to the ground state of the system is the so-called "Thomas-Fermi approximation" (TFA), which ignores the kinetic term in (5). The validity of the Gaussian distribution upon the increase of $g$ can be sorted out as follows. First, let's rescale the GP energy functional: $E /(\hbar \omega) \rightarrow E, t \omega \rightarrow t, r / \ell \rightarrow r$ with $\ell \equiv \sqrt{\hbar / m \omega}$ being the length scale throughout this paper. Next, divide the energy functional by $N$ (total number of particles), Eq. (5) for the current one-component system then becomes

$$
E / N=\int d \mathbf{r} \Phi^{*}\left[-\frac{1}{2} \nabla^{2}+\frac{1}{2} r^{2}+\frac{m}{2 \ell} N g|\Phi|^{2}\right] \Phi .
$$

By writing this way, it is seen clearly that in addition to $g$, both the magnetic trap potential (through $\ell$ ) and $N$ are also coupled to the nonlinear term. As a consequence, whether a Gaussian is a good approximation will depend on the magnitude of the front factor for the nonlinear term, $\mathrm{mNg} / 2 \ell \propto \mathrm{Ngm}^{3 / 2} \sqrt{\omega}$. Provided that $g$ is fixed, the smaller the value of $N \sqrt{\omega}$, the better the Gaussian distribution is for the same atoms.

In current experiments performed on the singlecomponent system, nonlinear effect is typically not small. Application of the Gaussian distribution to a real system thus always poses a question mark. Moreover, in a system of two-component mixture, the phenomenon "phase separation" could exist when the interspecies coupling $g_{12}$ is strong. In this case, using a Gaussian to describe the distribution of the individual component will be fundamentally ineffective.

\section{B. Modified Gaussian Distribution}

To better describe the system and for capturing the advantage of easy integration of the Gaussian function, we propose the following "modified Gaussian distribution":

$$
\begin{aligned}
\Phi(\mathbf{r}) & =C\left\{\exp \left[-\frac{A\left(r-r_{0}\right)^{2}}{2}\right]+\exp \left[-\frac{A\left(r+r_{0}\right)^{2}}{2}\right]\right\} \\
& =C^{\prime} \exp \left(-\frac{A r^{2}}{2}\right) \cosh \left(A r_{0} r\right),
\end{aligned}
$$

which is the superposition of two Gaussian functions, with centers deviated from the origin by $r_{0}$ and $-r_{0}$ respectively. As written explicitly in the second line of (12), the resulting function is a Gaussian function modified (multiplied) by a hypercosine function. In (12), $C$ and $C^{\prime}=2 C \exp \left(-A r_{0}^{2} / 2\right)$ are constants for normalization. $A$ and $r_{0}$ are parameters corresponding to the amplitude and overall density profile of the atom cloud. For a given $C$, the values of $A$ and $r_{0}$ are determined upon minimizing the ground-state energy $E$ of the system. In Fig. 1, $r_{0}$ dependence of the normalized density profile $|\Phi(\mathbf{r})|^{2} r^{2}$ is shown with a fixed $A$. As can be seen clearly, the maximum of $|\Phi(\mathbf{r})|^{2} r^{2}$ has a tendency to move outwards

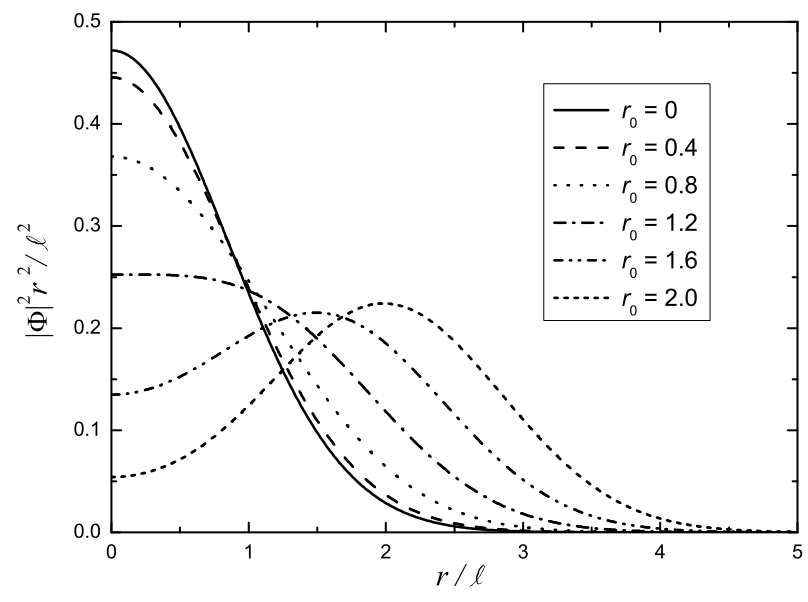

FIG. 1: The normalized density profile, $|\Phi(\mathbf{r})|^{2} r^{2}$, with $\Phi$ given by the modified Gaussian function in (12). Here $A=0.8$ is fixed for all cases.

when $r_{0}$ is increased. When $r_{0}$ is large, the maximum could move significantly away from the origin. Having this feature, which is important when phase separation occurs, the modified Gaussian function is considered to be more effective for a two-component mixture.

Compared to the simple Gaussian distribution [Eq. (10)], the modified Gaussian consists one more parameter $\left(r_{0}\right)$, that allows for a better description of the ground-state equilibrium properties of the system. As an illustration, in Fig. 2 we plot the ground-state energy $E$ [Eq. (11)] as a function of the scattering length $a$ for the modified Gaussian distribution. The parameters are taken to be: $\omega=2 \pi \times 160 \mathrm{~Hz}$ and $N=10^{4}$. Similar results for the simple Gaussian distribution and the TFA are also shown for comparison. In the small- $a$ regime (small nonlinear effect), the curve of modified Gaussian merges to the one of simple Gaussian, as it must be. Gaussian distribution is exact in the $a \rightarrow 0$ limit. The ground-state energy $E$ is seen to be much underestimated for TFA at small $a$. In the intermediate $a(=2 \sim 5)$ regime, nonlinear effect becomes more and more important, the curve of modified Gaussian starts to deviate (becomes lower) from the one of a simple Gaussian. This indicates that the modified Gaussian distribution is more favored by the system so long as the ground-state energy is concerned. It is worth noting that most current experiments lie in this intermediate $a$ regime. In the regime of large $a$, the results of TFA should be the most accurate ones.

In Fig. 31 spatial distributions (density profile) of the three cases (Gaussian, modified Gaussian, and TFA) are compared for different total numbers of particle $N$. The case of larger $N$ corresponds to the case of larger nonlinear effect. In frame (a) of smaller $N$, the curve of modified Gaussian is closer to the one of simple Gaussian - with the maximum at the origin. When $N$ is larger 


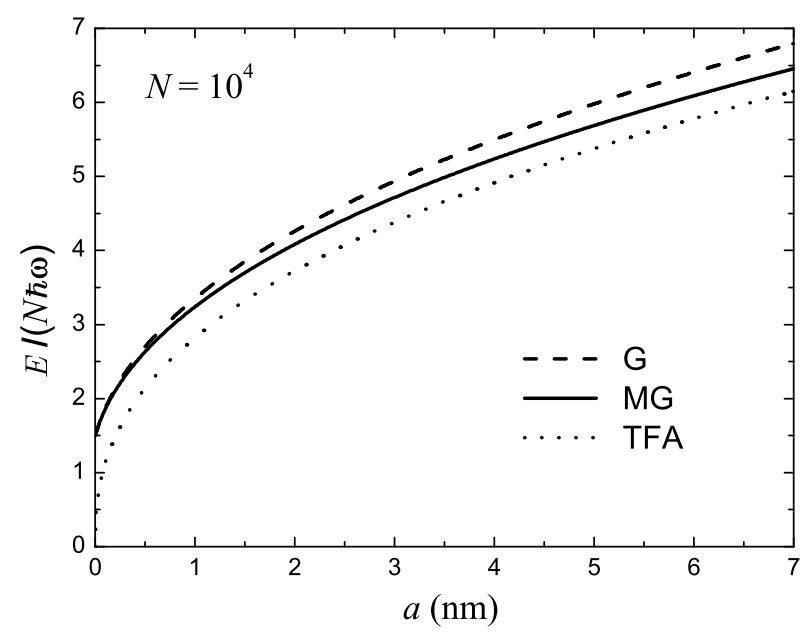

FIG. 2: Ground-state energy $E$ divided by $\hbar \omega$ ( $\omega=$ trap frequency) versus the scattering length $a$ for single-component condensed bosons. The curves are for Gaussian $(\mathrm{G})$, modified Gaussian (MG), and TFA. The parameters are $\omega=2 \pi \times$ $160 \mathrm{~Hz}$ and $N=10^{4}$.

[frame (b) \& (c)], the curves of modified Gaussian behave closer to the ones of TFA, although the maxima may deviate from the origin. As far as a real distribution is concerned, this feature is somewhat defective. It should be emphasized, however, that modified Gaussian has an overall distribution which is closer to the one of TFA and is more accurate for large nonlinear effect. Moreover, modified Gaussian inherits the advantage of easy integration of simple Gaussian, which makes variational method feasible for the problems.

In next section, equilibrium property and dynamics of a two-component system are studied. Breathing modes of such system will be shown to depend crucially on whether the system is miscible or phase-separated.

\section{TWO-COMPONENT MIXTURE}

The analyses in previous section are limited to singlecomponent systems. In the case of two-component mixture, the system could undergo a phase transition from a miscible state to a phase-separated state when the interspecies coupling is strong. For current inhomogeneous but isotropic system, when phase separation exists, one component of the atoms will have maximum density deviated from the origin, while the density of the other component remains maximum at the origin. As pointed out before, a simple Gaussian is ineffective to describe such phenomenon. The present section is devoted to study how the phase separation occurs when the modified Gaussian distributions are in effect. At the same time, the dynamics (breathing modes) of the two-component system will also be studied starting from the same modified Gaussian distribution.

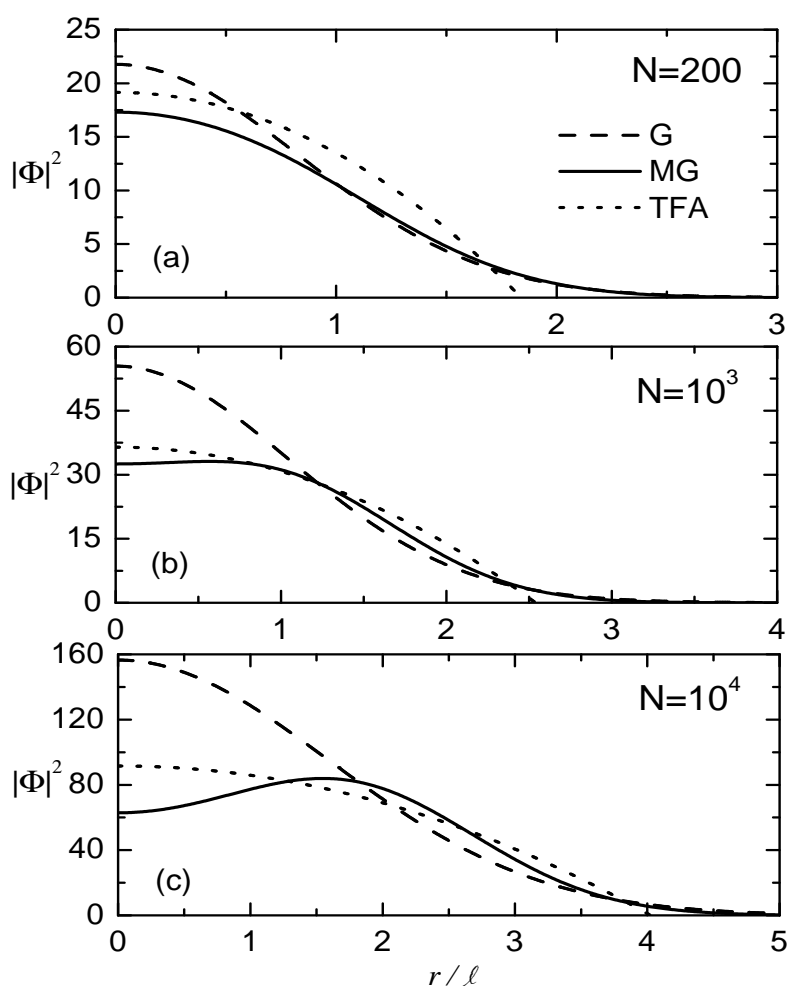

FIG. 3: The spatial dependence of the density profile $|\Phi(\mathbf{r})|^{2}$ for a one-component system. Three kinds of distribution (Gaussian, modified Gaussian, and TFA) are compared. Frame (a)-(c) are for number of atoms $N=200,10^{3}$, and $10^{4}$ respectively.

\section{A. Equilibrium Property}

The ground-state properties of the two-component bosonic atoms are first studied. Considering that both components are described by the modified Gaussian distribution

$$
\Phi_{i}(\mathbf{r})=C_{i} \exp \left(-\frac{1}{2} A_{i} r^{2}\right) \cosh \left(A_{i} r_{i} r\right)
$$

where $i=1,2$ and the normalization factor $C_{i}$ is dependent of the total number $N_{i}$ of individual component $i$. The ground-state energy $E$ of the system can be calculated by substitution of (13) into Eqs. (11)-(6) and (8). Given three coupling strength $a_{1}, a_{2}$, and $a_{12}$, and the particle number $N_{1}$ and $N_{2}$, final result of $E$ can be obtained by minimizing $E$ against the variation of $A_{1}$ and

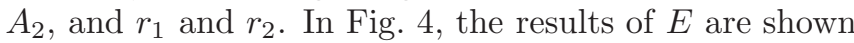
as a function of the inter-component scattering length $a_{12}$. Two cases of equal population $N_{1}=N_{2} \equiv N=10^{3}$ and $N=10^{4}$ are presented. The slopes, $\partial E / \partial a_{12}$ vs. $a_{12}$ are also shown. For easy comparison, throughout this section the parameters are taken to be the same as those in Ref. 19]. Based on the experiment of $\mathrm{Na}-\mathrm{Rb}$ mixture, the scattering lengths of $\mathrm{Na}$ and $\mathrm{Rb}$ atoms are chosen to be $3 \mathrm{~nm}$ and $6 \mathrm{~nm}$ respectively. Regarding the 

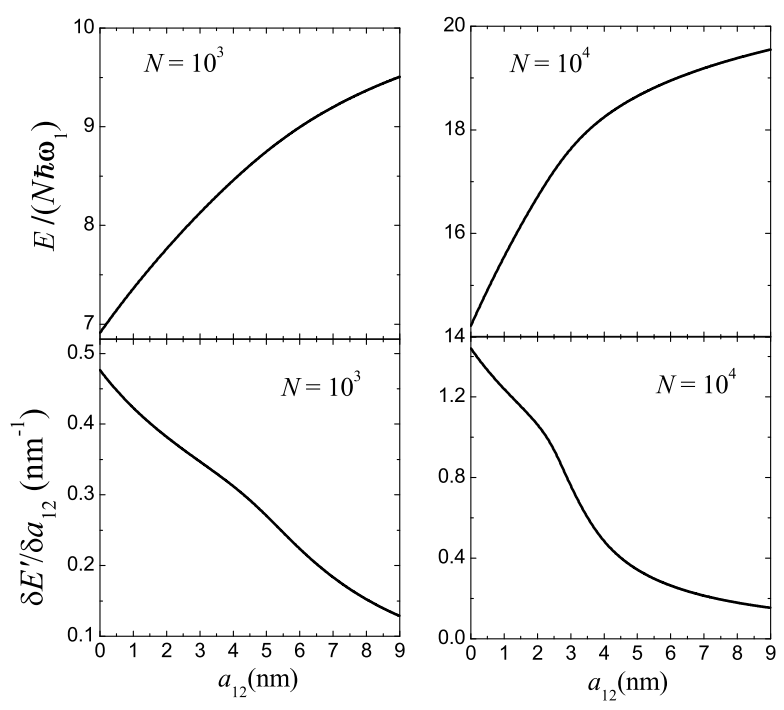

FIG. 4: The dependence of inter-component scattering length $a_{12}$ on the ground-state energy $E$, divided by $N \hbar \omega_{1}$, of a twocomponent Boson system. Modified Gaussian distribution is undertaken. The slopes of $\partial E / \partial a_{12}$ vs. $a_{12}$ are also shown $\left[E^{\prime} \equiv E /\left(N \hbar \omega_{1}\right)\right]$. Left column corresponds to $N_{1}=N_{2} \equiv$ $N=10^{3}$, while right column corresponds to $N=10^{4}$. See text for the values of other parameters.

external magnetic trap, the corresponding trap frequencies are $\omega_{1}=2 \pi \times 310 \mathrm{~Hz}$ for $\mathrm{Na}$ and $\omega_{2}=2 \pi \times 160 \mathrm{~Hz}$ for $\mathrm{Rb}$ respectively. In both plots in Fig. 4 (especially $\partial E / \partial a_{12}$ vs. $\left.a_{12}\right)$, one sees clear evidence that the system undergoes a transition from one phase to another at $a_{12} \sim 2-2.5$ when $N=10^{4}$ and at $a_{12} \sim 4-5$ when $N=10^{3}$.

Fig. 5 shows how this transition behaves in the density profiles of the two-component system. The figures are plotted in a variety of interspecies scattering lengths $\left(a_{12}\right)$ with fixed $a_{1}=3 \mathrm{~nm}$ and $a_{2}=6 \mathrm{~nm}$. In the case of $N=10^{4}$, it is found that both $\left|\Phi_{1}(r)\right|^{2}$ and $\left|\Phi_{2}(r)\right|^{2}$ have maximum away from the center when $a_{12}=0$. It has to be emphasized again, that modified Gaussian distribution has a feature that the maximum density could deviate from the origin due to large nonlinear effect. Even though $a_{12}=0$, the individual nonlinear effect is still large, which lead to such "unphysical" result. As mentioned before, it is the fact that modified Gaussian has an overall distribution more close to TFA, that one has to look into. When $a_{12}$ becomes larger $\left(a_{12} \sim 2-2.5\right)$, the maximum of $\left|\Phi_{1}(r)\right|^{2}$ is pushed significantly away from the center, while the maximum of $\left|\Phi_{2}(r)\right|^{2}$ is right at the center - the pheomenon of phase separation.

To see this phenomenon more clearly, in Fig. 6 we plot the value of parameter $r_{1}$ (for the modified Gaussian) versus $a_{12}$. It is reminded that $r_{1}$ is referred to the $\mathrm{Na}$ component in Fig. 5. In the case of $N=10^{4}$, there is a sudden change for $r_{1}$ at $a_{12} \sim 2-2.5$. This is in great consistence with the ground-state energy shown in Fig. 4 where a sharp change also occurs at this range of $a_{12}$. In view of the original definition of $r_{1}$ in the
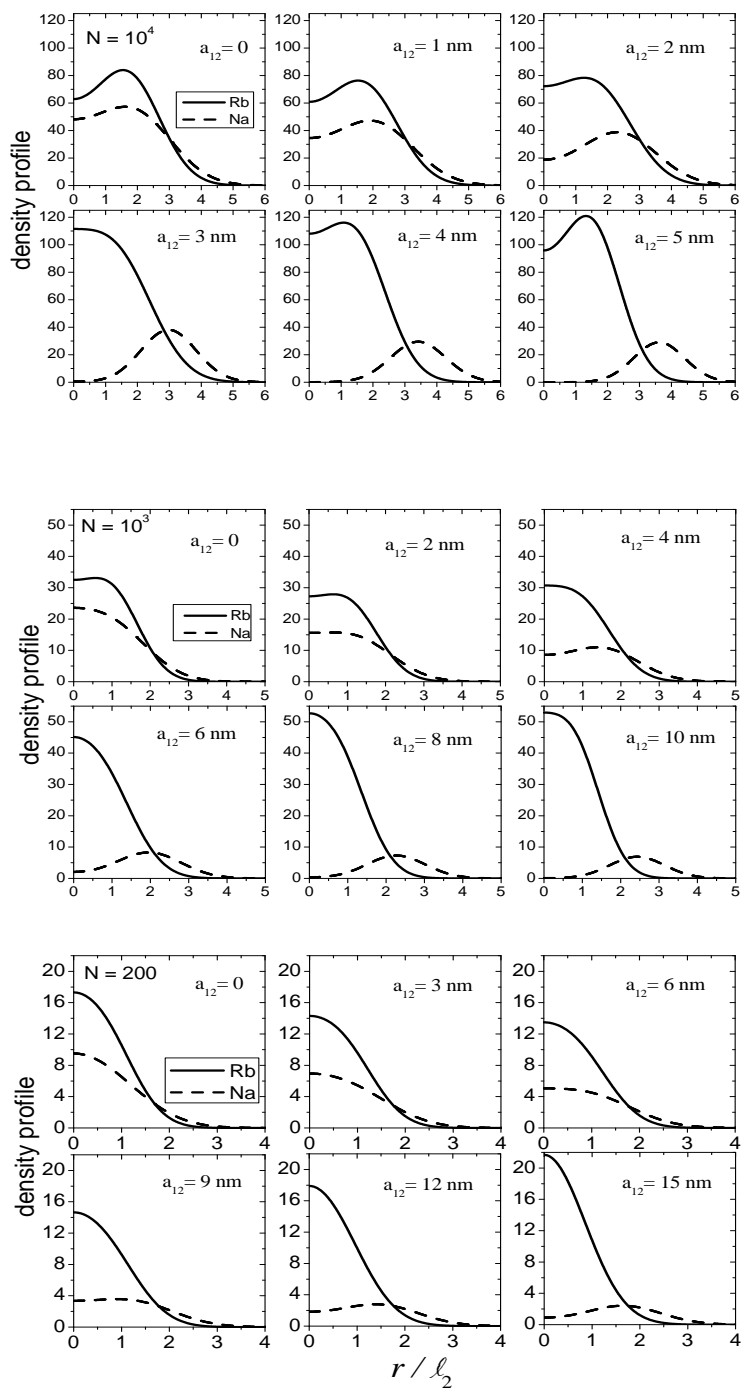

FIG. 5: The individual density profile plotted in a variety of the interspecies scattering length $a_{12}$ for a two-component system. From the top to the bottom are for $N_{1}=N_{2} \equiv N=$ $10^{4}, 10^{3}$ and 200 respectively. Note that in $x$ axis, $r$ is scaled to $\ell_{2}=\sqrt{\hbar / m_{2} \omega_{2}}$.

first line of Eq. (12) (i.e., $r_{0}$ there), a sudden change of $r_{1}$ means a sudden change of the coordinate of density maximum (see also Fig. 1). This sudden change indeed corresponds to the transition of "phase separation". In the case of $N=10^{3}$, we also see a consistent picture among the results in Figs. 46 6 . A phase separation occurs at $a_{12} \sim 4-5$. When $N=200$, the phenomenon is less obvious. However, one can still see a phase separation that appears at $a_{12} \sim 9-10$.

\section{B. Breathing Modes}

In this section, we investigate how the phase separation affects the dynamics of the two-component system. 


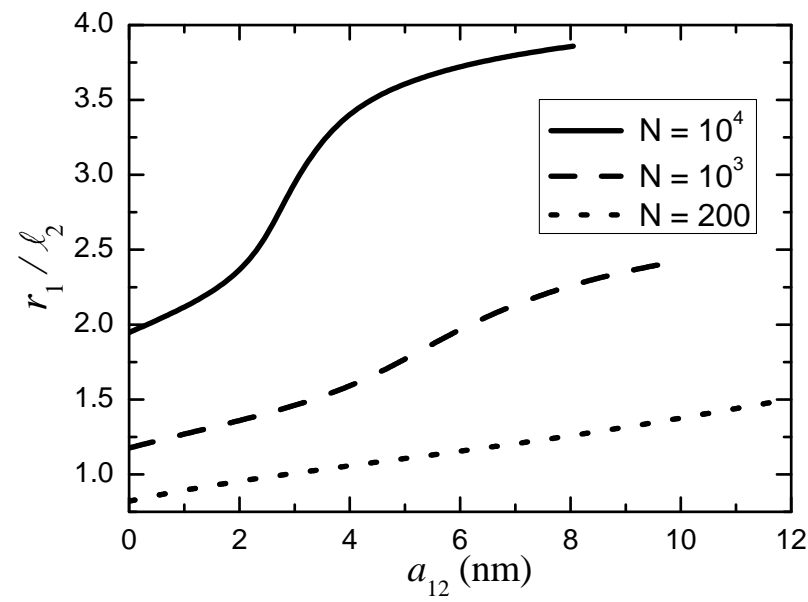

FIG. 6: The value of the parameter $r_{1}$ divided by $\ell_{2}\left(r_{1}\right.$ is referred to $\mathrm{Na}$ component in Fig. 5) for the modified Gaussian plotted as a function of the interspecies scattering length $a_{12}$.

It was shown in Ref. [19] that for a two-component mixture, the collective mode dispersion will have a drastic change near the occurrence of phase separation. Since the property of collective mode is intimately connected to the equilibrium property of the system, the sharp transition in the ground-state energy of the system could imply a drastic collective mode dispersion change in the same regime.

In the current context, we will focus on the breathing modes of the system. One first extends the modified Gaussian distribution for each component [Eq. (13)] to include the dynamical variables [24, 25]:

$$
\begin{aligned}
\Phi_{i}(\mathbf{r}, t)= & C_{i} \exp \left\{-\frac{1}{2} A_{i}\left[1+\varepsilon_{i}(t)+i \varepsilon_{i}^{\prime}(t)\right] r^{2}\right\} \\
& \times \cosh \left(A_{i} r_{i} r\right) .
\end{aligned}
$$

Here $\varepsilon_{i}$ and $\varepsilon_{i}^{\prime}$ correspond to the fluctuations of local amplitude and local phase of the atom cloud along the radial $r$ direction. $A_{i}$ and $r_{i}$ are determined earlier through minimizing the ground-state energy of the system (see previous subsection). Since we are interested in the breathing modes of the lowest energy and of only one radial node $(n=1), \varepsilon_{i}$ and $\varepsilon_{i}^{\prime}$ are coupled only to the $r^{2}$ term in the Gaussain function in (14). One may introduce extra dynamical variables to the hypercosine function in (14), that could lead to more branches of radial modes. Some of them will have more than one node $(n \geq 2)$.

Substitution of the above dynamical wave function into Eqs. (77) and (8), one can derive the coupled equations of motion [using (9)] for $\varepsilon_{i}$ and $\varepsilon_{i}^{\prime}$, and then solve a corresponding dynamical matrix to obtain the dispersions of relations for the breathing modes. In Fig. 7, we present the breathing mode dispersion as a function of the inter-

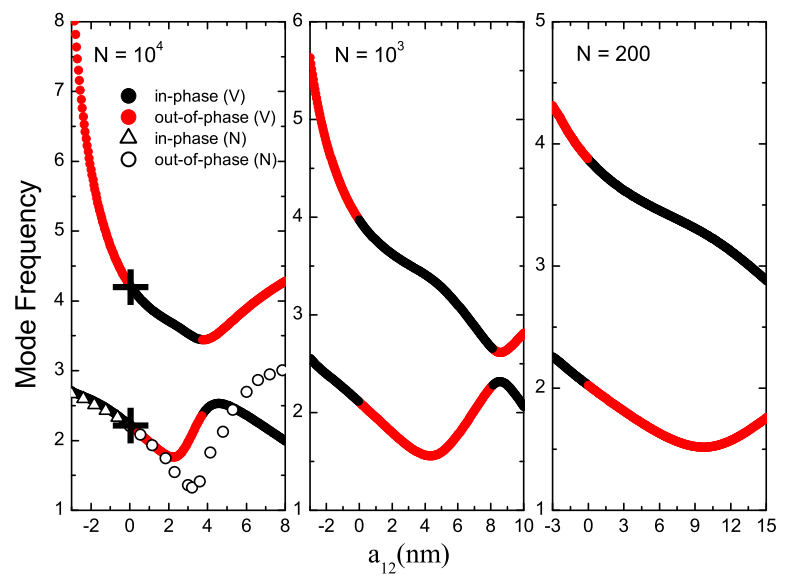

FIG. 7: (Color online) Based on the variational method (V), in-phase and out-of-phase breathing mode dispersions are plotted as a function of $a_{12}$ for a two-component boson system. In each frame, first turning point in lower curves corresponds to where the system starts to be phase separated. The second turning point corresponds to the exchange of the inphase and out-of-phase modes. In the left frame of $N=10^{4}$, the results given by numerical calculation [19] (N) are compared. The two crosses at $a_{12}=0$ correspond to the breathing modes of individual component 1 and 2 (decoupled regime).

component scattering length $a_{12}$. Again, all parameters are the same as before. In the case of $N=10^{4}$, the results given by numerical calculation in Ref. 19] is included for comparison.

Much information is revealed in Fig. 7. Taking the $N=10^{4}$ case as an example, when interspecies scattering length $a_{12}$ is negative, the system is miscible. In-phase breathing mode has lower energy and is more easily excited. When $a_{12}$ is positive, the results are divided into three regimes. When $0 \leq a_{12} \lesssim 2.5$, the system is miscible and thus out-of-phase breathing mode has lower energy and more easily gets excited. When the system starts to phase separate but most parts of the two components are still overlapping $\left(2.5 \lesssim a_{12} \lesssim 4\right)$, the energy of the out-of-phase breathing mode remains lower than that of in-phase mode. When the two components are well separated (not much overlap) at $a_{12} \gtrsim 4$ (see also Fig. 5), in-phase breathing mode turns out to have lower energy again. The switch of the in-phase and out-ofphase breathing modes in the above latter two cases can be understood alternatively as follows. When phase separation occurs but the two components are still very much overlapped, out-of-phase mode is more easily excited because it corresponds to the decrease of the overlap (and the energy). However, when the two components are well separated, in-phase mode is in turn more easily excited because in this case out-of-phase mode simply increases the overlap (and the energy).

As seen in Fig. 7(a), there are two turning points in the lower dispersion curve. The first turning point is the signature of the system being phase separated. While 
the second turning point corresponds to the exchange of the in-phase and out-of-phase breathing modes in the regime where the two components are well separated. In comparison with the numerical results given by Ref. [19] (where only the lowest-energy mode is given), our result of lowest-energy mode matches theirs quantitatively before phase separation arises. After the phase separation occurs, our result is qualitatively similar to their. (There is no second turning point seen in Ref. [19] for the range plotted.) It is worth noting that the two points of $a_{12}=0$ correspond actually to the breathing modes of two decoupled components (lower-frequency one is for $\mathrm{Rb}$, while higher-frequency one is for Na). From the goodness of the match of our results to those of Ref. [19], it strongly suggests that modify Gaussian is also a good function to describe the dynamics of a one-component system when the nonlinear effect is important.

\section{CONCLUSIONS}

In conclusion, the density profile, the ground-state energy, and the breathing modes of a two-component
Bose condensed system are studied using a variational method. We propose a modified Gaussian distribution function which is shown to be more effective for a twocomponent system. While the modified Gaussian function reserves the advantage of easy integration with the simple Gaussian, it actually gives a better description even to the equilibrium and dynamical properties of a single-component system. Our approach can be generalized to study the vortices of a two-component system, and possibly the system of imbalanced spin population.

\section{Acknowledgments}

We acknowledge fruitful discussions with Daw-Wei Wang. This work is supported by National Science Council of Taiwan under Grant No. 94-2112-M-003-011. We also acknowledge the support from the National Center for Theoretical Sciences, Taiwan.
[1] C. J. Myatt, E. A. Burt, R. W. Ghrist, E. A. Cornell, and C. E. Wieman, Phys. Rev. Lett. 78, 586 (1997).

[2] D. S. Hall, M. R. Matthews, J. R. Ensher, C. E. Wieman, and E. A. Cornell, Phys. Rev. Lett. 81, 1539 (1998).

[3] G. Modugno, G. Ferrari, G. Roati, R. J. Brecha, A. Simoni, M. Inguscio, Science 294, 1320 (2001).

[4] M. Anderlini, E. Courtade, M. Cristiani, D. Cossart, D. Ciampini, C. Sias, O. Morsch, and E. Arimondo, Phys. Rev. A 71, 061401 (2005).

[5] A.G. Truscott, K.E. Strecker, W.I. McAlexander, G.B. Partridge, and R.G. Hulet, Science 291, 2570 (2001).

[6] F. Schreck, L. Khaykovich, K.L. Corwin, G. Ferrari, T. Bourdel,J. Cubizolles, and C. Salomon, Phys. Rev. Lett. 87, 080403 (2001).

[7] Z. Hadzibabic, C. A. Stan, K. Dieckmann, S. Gupta, M. W. Zwierlein, A. Görlitz, and W. Ketterle, Phys. Rev. Lett. 88, 160401 (2002).

[8] J. Goldwin, S.B. Papp, B. DeMarco, and D.S. Jin, Phys. Rev. A 65, 021402 (2002).

[9] C. A. Regal, C. Ticknor, J. L. Bohn, D. S. Jin, Nature 424, 47 (2003).

[10] S. Jochim et al., Science 302, 2101 (2003).

[11] K. E. Strecker, G. B. Partridge, and R.G. Hulet, Phys. Rev. Lett. 91, 080406 (2003).

[12] C. Chin et al., Science 305, 1128 (2004).

[13] G. B. Partridge, K. E. Strecker, R. I. Kamar, M. W.
Jack,R. G. Hulet, Phys. Rev. Lett 95, 020404 (2005).

[14] M. W. Zwierlein, J. R. Abo-Shaeer, A. Schirotzek,C. H. Schunck, W. Ketterle, Nature 435, 1047 (2005).

[15] M. W. Zwierlein, A. Schirotzek, C. H. Schunck, and W. Ketterle, Science 311, 492 (2006).

[16] G. B. Partridge, W. Li, R. I. Kamar, Y. Liao, R. G. Hulet, Science 311, 503 (2006).

[17] T.-L. Ho and V.B. Shenoy, Phys. Rev. Lett. 77, 3276 (1996).

[18] E.P. Bashkin and A.V. Vagov, Phys. Rev. B 56, 6207 (1997).

[19] H. Pu and N.P. Bigelow, Phys. Rev. Lett. 80, 1130 (1998).

[20] K. Mølmer, Phys. Rev. Lett. 80, 1804 (1998).

[21] N. Nygaard and K. Molmer, Phys. Rev. A 59, 2974 (1999).

[22] J. Kinnunen, L. M. Jensen, and P. Törmä, Phys. Rev. Lett. 96, 110403 (2006).

[23] P. Pieri and G. C. Strinati, Phys. Rev. Lett 96, 150404 (2006).

[24] J.-P. Martikainen and H. T. C. Stoof, Phys. Rev. A 69, 23608 (2004).

[25] C.-C. Huang and W. C. Wu, Phys. Rev. A 72, 065601 (2005) 